\title{
A Struwe Type Decomposition Result for a Singular Elliptic Equation on Compact Riemannian Manifolds
}

\author{
Youssef Maliki ${ }^{1, *}$ and Fatima Zohra Terki ${ }^{2}$ \\ 1 Department of Mathematics, University Aboubekr Belkaid of Tlemcen, Tlemcen, \\ Algeria \\ 2 Higher School in Business and Management, Tlemcen, Algeria
}

Received 21 February 2017; Accepted (in revised version) 31 August 2017

Abstract. On a compact Riemannian manifold, we prove a decomposition theorem for arbitrarily bounded energy sequence of solutions of a singular elliptic equation.

Key Words: Yamabe equation, critical Sobolev exponent, Hardy inequality, bubbles.

AMS Subject Classifications: 58J05

\section{Introduction}

Let $(M, g)$ be an $(n \geq 3)$-dimensional Riemannian manifold. In this paper, we are interested in studying on $(M, g)$ the asymptotic behaviour of a sequence of solutions $u_{\alpha}$, when $\alpha \rightarrow \infty$, of the following singular elliptic equation:

$$
\Delta_{g} u-\frac{h_{\alpha}}{\rho_{p}^{2}(x)} u=f(x)|u|^{2^{*}-2} u,
$$

where $2^{*}=\frac{2 n}{n-2}, h_{\alpha}$ and $f$ are functions on $M, p$ is a fixed point of $M$ and $\rho_{p}(x)=\operatorname{dist}_{g}(p, x)$ is the distance function on $M$ based at $p$ (see Definition 2.2).

Certainly, if the singular term $\frac{h_{\alpha}}{\rho_{p}^{2}(x)}$ is replaced by $\frac{n-2}{4(n-1)} S c a l_{g}$, then equation $E_{\alpha}$ becomes the prescribed scalar curvature equation which is very known in the literature. When $f$ is constant and the function $\rho_{p}$ is of power $0<\gamma<2$, Eq. $\left(E_{\alpha}\right)$ can be seen as a case of equations that arise in the study of conformal deformation to constant scalar curvature of metrics which are smooth only in some ball $B_{p}(\delta)$ (see [5]).

${ }^{*}$ Corresponding author. Email addresses: malyouc@yahoo.fr (Y. Maliki), fatimazohra113@yahoo.fr (F. Z. Terki) 
Equations of type $\left(E_{\alpha}\right)$ have been the subject of interest especially on the Euclidean space $I R^{n}$. Let $D^{1,2}\left(I R^{n}\right)$ be the Sobolev space defined as the completion of $C_{0}^{\infty}\left(I R^{n}\right)$, the space of smooth functions with compact support in $I R^{n}$, with respect to the norm

$$
\|u\|_{D^{1,2}\left(I R^{n}\right)}^{2}=\int_{I^{n}}|\nabla u|^{2} d x .
$$

A famous result has been obtained in [8] and it consists of the classification of positive solutions $u \in D^{1,2}\left(I R^{n}\right)$ of the equation

$$
\Delta u-\frac{\lambda}{|x|^{2}} u=u^{\frac{n+2}{n-2}}
$$

where $0<\lambda<\frac{(n-4)^{2}}{4}$, into the family of functions

$$
u_{\lambda}(x)=C_{\lambda}\left(\frac{|x|^{a-1}}{1+|x|^{2 a}}\right)^{\frac{n}{2}-1}
$$

where $C_{\lambda}$ is some constant and $a=\sqrt{1-\frac{4 \lambda}{(n-2)^{2}}}$.

In terms of decomposition of Palais-Smale sequences of the functional energy, this family of solutions was employed in [6] to construct singular bubbles,

$$
\mathcal{B}_{\lambda}^{\varepsilon_{\alpha}, y_{\alpha}}=\varepsilon_{\alpha}^{\frac{2-n}{2}} u_{\lambda}\left(\frac{x-y_{\alpha}}{\varepsilon_{\alpha}}\right) \quad \text { with } \frac{\left|y_{\alpha}\right|}{\varepsilon_{\alpha}} \rightarrow 0,
$$

which, together with the classical bubbles caused by the existence of critical exponent

$$
\mathcal{B}_{0}^{\varepsilon_{\alpha}, y_{\alpha}}=\varepsilon_{\alpha}^{\frac{2-n}{2}} u_{0}\left(\frac{x-y_{\alpha}}{\varepsilon_{\alpha}}\right) \quad \text { with } \frac{\left|y_{\alpha}\right|}{\varepsilon_{\alpha}} \rightarrow \infty,
$$

where $u_{0}$ being the solution of the non perturbed equation $\Delta u=u^{\frac{n+2}{n-2}}$, give a whole picture of the decomposition of the Palaise-Smale sequences. This decomposition result has been proved in [6] and was the key component for the obtention of interesting existence results for Eq. $(E)$ with a function $K$ get involved in the nonlinear term. Similar decomposition result has been obtained in [1] for Eq. $(E)$ with small perturbation, the authors described asymptotically the associated Palais-Smale sequences of bounded energy.

The compactness result obtained in this paper can be seen as an extension to Riemannian context of those obtained in [6] and [1] in the Euclidean context, the difficulties when working in the Riemannian setting reside mainly in the construction of bubbles.

Historically, a famous compactness result for elliptic value problems on domains of $\mathbb{R}^{n}$ has been obtained by M. Struwe in [7]. Struwe's result has been extended later by O. Druet et al. in [2] to elliptic equations on Riemannian manifolds in the form

$$
\Delta_{g} u+h_{\alpha} u=u^{2^{*}-1} .
$$


Many results have been obtained by the authors describing the asymptotic behaviour of Palais-Smale sequences. The authors gave a detailed construction of bubbles by means of a re-scaling process via the exponential map at some points, supposed to be the centers of bubbles. The author in [3] followed the same procedure to prove a decomposition result on compact Riemannian manifolds for a Sobolev-Poincaré equation.

In this paper, we follow closely the work in [2] to prove a decomposition theorem for Eq. $\left(E_{\alpha}\right)$. More explicitly, after determining conditions under which solutions of $\left(E_{\alpha}\right)$ exist, we prove as in [6] and [1] that, under some conditions on the sequence $h_{\alpha}$ and the function $f$, a sequence of solutions of $\left(E_{\alpha}\right)$ of arbitrarily bounded energy decomposes into the sum of a solution of the the limiting equation

$$
\Delta_{g} u-\frac{h_{\infty}(p)}{\rho_{p}^{2}(x)} u=f(p)|u|^{2^{*}-2} u
$$

where $h_{\infty}$ is the uniform limit of $h_{\alpha}$, and two kinds of bubbles, namely the classical and the singular ones due to the presence respectively of the critical exponent and the singular term.

\section{Notations and preliminaries}

In this section, we introduce some notations and materials necessary in our study. Let $H_{1}^{2}(M)$ be the Sobolev space consisting of the completion of $\mathcal{C}^{\infty}(M)$ with respect to the norm

$$
\|u\|_{H_{1}^{2}(M)}^{2}=\int_{M}\left(|\nabla u|^{2}+u^{2}\right) d v_{g} .
$$

$M$ being compact, $H_{1}^{2}(M)$ is then embedded in $L_{q}(M)$ compactly for $q<2^{*}=\frac{2 n}{n-2}$ and continuously for $q=2^{*}$.

Let $K(n, 2)$ denote the best constant in Sobolev inequality that asserts that there exists a constant $B>0$ such that for any $u \in H_{1}^{2}(M)$,

$$
\|u\|_{L_{2^{*}}(M)}^{2} \leq K^{2}(n, 2)\|\nabla u\|_{L_{2}(M)}^{2}+B\|u\|_{L_{2}(M)}^{2} .
$$

Throughout the paper, we will denote by $B(a, r)$ a ball of center $a$ and radius $r>0$, the point $a$ will be specified either in $M$ or in $I R^{n}$, and $B(r)$ is a ball in $I R^{n}$ of center 0 and radius $r>0$.

Denote by $\delta_{g}$ the injectivity radius of $M$. Let $p \in M$ be a fixed point, as in [5] we define the function $\rho_{p}$ on $M$ by

$$
\rho_{p}(x)= \begin{cases}\operatorname{dist}_{g}(p, x), & \operatorname{dist}_{g}(p, x)<\delta_{g} \\ \delta_{g}, & \operatorname{dist}_{g}(p, x) \geq \delta_{g}\end{cases}
$$

For $q \geq 1$, we denote by $L_{q}\left(M, \rho_{p}^{2}\right)$ the space of functions $u$ such that

$$
\int_{M} \rho_{p}^{2}|u|^{q} d v_{g}<\infty
$$


This space is endowed with norm

$$
\|u\|_{q, \rho_{p}^{\theta}}^{q}=\int_{M} \rho_{p}^{\theta}|u|^{q} d v_{g}
$$

In [5], the following Hardy inequality has been proven on any compact manifold $M$, for every $\varepsilon>0$ there exists a positive constant $A(\varepsilon)$ such that for any $u \in H_{1}^{2}(M)$,

$$
\int_{M} \frac{u^{2}}{\rho_{p}^{2}} d v_{g} \leq\left(K^{2}(n, 2,-2)+\varepsilon\right) \int_{M}|\nabla u|^{2} d v_{g}+A(\varepsilon) \int_{M} u^{2} d v_{g},
$$

with $K(n, 2,-2)$ being the best constant in the Euclidean Hardy inequality

$$
\int_{\mathbb{R}^{n}} \frac{u^{2}}{|x|^{2}} d x \leq K(n, 2,-2)^{2} \int_{\mathbb{R}^{n}}|\nabla u|^{2} d x, u \in \mathcal{C}_{o}^{\infty}\left(\mathbb{R}^{n}\right) .
$$

If $u$ is supported in a ball $B(p, \delta), 0<2 \delta<\delta_{g}$, then

$$
\int_{B(p, \delta)} \frac{u^{2}}{\rho_{p}^{2}} d v_{g} \leq K_{\delta}(n, 2,-2) \int_{B(p, \delta)}|\nabla u|^{2} d v_{g}
$$

with $K_{\delta}(n, 2,-2)$ goes to $K(n, 2,-2)$ when $\delta$ goes to 0 .

Concerning the existence of solutions of Eqs. $\left(E_{\alpha}\right)$, the author in [5] proved through the classical variational techniques an existence result with $f$ a constant function. Following closely the strategy in [5], we obtain the existence of a weak solution $u_{\alpha}$ of the Eq. $\left(E_{\alpha}\right)$. This existence result is formulated in the following theorem and due to the very familiarity of the techniques used, in order to avoid heaviness in the paper, we omit the proof (for a good presentation of these techniques, see for example [4]). For $u \in H_{1}^{2}(M)$, set

$$
\mu=\inf _{u \in H_{1}^{2}(M), u \neq 0} \frac{\int_{M}\left(|\nabla u|^{2}-\frac{h}{\rho_{p}^{2}} u^{2}\right) d v_{g}}{\left(\int_{M} f|u|^{2} d v_{g}\right)^{\frac{2}{2^{*}}}} .
$$

The following theorem ensures conditions under which a weak solution $u_{\alpha}$ of $\left(E_{\alpha}\right)$ exists.

Theorem 2.1. Let $(M, g)$ be a compact $n(n \geq 3)$-dimensional Riemannian manifold and $f, h_{\alpha}$ $(\alpha \in[0, \infty])$ be continuous functions on $M$. Under the following conditions:

1. $0<h_{\alpha}(p)<\frac{1}{K^{2}(n, 2,-2)}$,

2. $f(x)>0, \forall x \in M$ and $\mu<\frac{1-h_{\alpha}(p) K^{2}(n, 2,-2)}{\left(\sup _{M} f\right)^{\frac{n-2}{n}} K^{2}(n, 2)}$,

Eq. $\left(E_{\alpha}\right)$ admits a nontrivial weak solution $u_{\alpha} \in H_{1}^{2}(M)$. 


\section{Decomposition theorem}

Let $J_{\alpha}$ be the functional defined on $H_{1}^{2}(M)$ by

$$
J_{\alpha}(u)=\frac{1}{2} \int_{M}\left(|\nabla u|^{2}-\frac{h_{\alpha}}{\rho^{2}} u^{2}\right) d v_{g}-\frac{1}{2^{*}} \int_{M} f|u|^{2^{*}} d v_{g} .
$$

Traditionally, we define a Palais-Smale sequence $v_{\alpha}$ of $J_{\alpha}$ at a level $\beta$ as to be the sequence that satisfies $J_{\alpha}\left(v_{\alpha}\right) \rightarrow \beta$ and $D J_{\alpha}\left(v_{\alpha}\right) \varphi \rightarrow 0, \forall \varphi \in H_{1}^{2}(M)$.

Define the following limiting functionals

$$
\begin{array}{ll}
J_{\infty}(u)=\frac{1}{2}\left(\int_{M}\left(|\nabla u|^{2}\right)-\frac{h_{\infty}}{\rho^{2}} u^{2}\right) d v_{g}-\frac{1}{2^{*}} \int_{M} f|u|^{2^{*}} d v_{g}, & u \in H_{1}^{2}(M), \\
G(u)=\frac{1}{2} \int_{I R^{n}}|\nabla u|^{2} d x-\frac{1}{2^{*}} \int_{I R^{n}}|u|^{2^{*}} d x, & u \in D^{1,2}\left(I^{n}\right), \\
G_{\infty}(u)=\frac{1}{2} \int_{I R^{n}}|\nabla u|^{2} d x-\frac{h_{\infty}(p)}{2} \int_{I R^{n}} \frac{u^{2}}{|x|^{2}} d x-\frac{f(p)}{2^{*}} \int_{I R^{n}}|u|^{2^{*}} d x, & u \in D^{1,2}\left(I^{n}\right) .
\end{array}
$$

For $\alpha \in[0, \infty]$, let $h_{\alpha}$ be a sequence of continuous functions on $M$ such that

$$
(\mathcal{H})\left\{\begin{array}{l}
\text { a- }\left|h_{\alpha}(x)\right| \leq C, \text { for some constant } C>0, \forall x \in M \text { and } \forall \alpha \in[0, \infty], \\
\text { b- There exists a function } h_{\infty} \text { such that } \sup _{M}\left|h_{\alpha}-h_{\infty}\right| \rightarrow 0, \text { as } \alpha \rightarrow \infty, \\
\text { c- } 0<h_{\alpha}(p)<\frac{1}{K^{2}(n, 2,-2)} \text { for all } \alpha, 0 \leq \alpha \leq \infty
\end{array}\right.
$$

Now, we state our main result

Theorem 3.1. Let $(M, g)$ be a compact Riemannian manifold with $\operatorname{dim}(M)=n \geq 3, h_{\alpha}$ be a sequence of continuous functions on $M$ satisfying $(\mathcal{H}), f$ be a positive continuous function on $M$ that satisfies with $h_{\alpha}$ the conditions of Theorem 2.1. Let $u_{\alpha}$ be a sequence of weak solutions of $\left(E_{\alpha}\right)$ such that $\int_{M} f\left|u_{\alpha}\right|^{2^{*}} d v_{g} \leq C, \forall \alpha>0$. Then, there exist $k \in I N$, sequences $R_{\alpha}^{i}>0, R_{\alpha}^{i} \underset{\alpha \rightarrow \infty}{\rightarrow} 0$, $l \in I N$ sequences $r_{\alpha}^{j}>0, r_{\alpha}^{j} \underset{\alpha \rightarrow \infty}{\rightarrow} 0$, converging sequences $x_{\alpha}^{j} \rightarrow x_{o}^{j} \neq p$ in $M$, a solution $u_{o} \in H_{1}^{2}(M)$ of $\left(E_{\infty}\right)$, solutions $v_{i} \in D^{1,2}\left(I^{n}\right)$ of (3.9) and nontrivial solutions $v_{j} \in D^{1,2}\left(I^{n}\right)$ of (3.14) such that up to a subsequence

$$
\begin{aligned}
u_{\alpha}= & u_{o}+\sum_{i=1}^{k}\left(R_{\alpha}^{i}\right)^{\frac{2-n}{n}} \eta_{\delta}\left(\exp _{p}^{-1}(x)\right) v_{i}\left(\left(R_{\alpha}^{i}\right)^{-1} \exp _{p}^{-1}(x)\right) \\
& +\sum_{j=1}^{l}\left(r_{\alpha}^{j}\right)^{\frac{2-n}{n}} f\left(x_{o}\right)^{\frac{2-n}{4}} \eta_{\delta}\left(\exp _{x_{\alpha}^{j}}^{-1}(x)\right) v_{j}\left(\left(r_{\alpha}^{j}\right)^{-1} \exp _{x_{\alpha}^{j}}^{-1}(x)\right)+\mathcal{W}_{\alpha}, \\
& \text { with } \mathcal{W}_{\alpha} \rightarrow 0 \text { in } H_{1}^{2}(M),
\end{aligned}
$$

and

$$
J_{\alpha}\left(u_{\alpha}\right)=J_{\infty}\left(u_{o}\right)+\sum_{i=1}^{k} G_{\infty}\left(v_{i}\right)+\sum_{j=1}^{l} f\left(x_{o}^{j}\right)^{\frac{2-n}{2}} G\left(v_{j}\right)+o(1) .
$$


In order to prove this theorem, we prove some useful lemmas. In all what follows, $h_{\alpha}$ is supposed to satisfy conditions $(\mathcal{H})$.

Lemma 3.1. Let $u_{\alpha}$ be a Palais-Smale sequence for $J_{\alpha}$ at level $\beta$ that converges to a function $u$ weakly in $H_{1}^{2}(M)$ and $L_{2}\left(M, \rho_{p}^{2}\right)$, strongly in $L_{q}(M), 1 \leq q<2^{*}$ and almost everywhere in $M$. Then, the sequence $v_{\alpha}=u_{\alpha}-u$ is sequence of Palais-Smale for $J_{\alpha}$ and

$$
J_{\alpha}\left(v_{\alpha}\right)=\beta-J_{\infty}(u)+o(1) .
$$

Proof. First, in view of the fact that $u_{\alpha}$ is a Palais-Smale sequence for $J_{\alpha}, u_{\alpha}$ is bounded in $H_{1}^{2}(M)$. In fact, $D J_{\alpha}\left(u_{\alpha}\right) u_{\alpha}=o\left(\|u\|_{H_{1}^{2}(M)}\right)$ implies that

$$
J_{\alpha}\left(u_{\alpha}\right)=\frac{1}{n} \int_{M} f\left|u_{\alpha}\right|^{2^{*}} d v_{g}=\beta+o(1)+o\left(\|u\|_{H_{1}^{2}(M)}\right) .
$$

Since $f>0$, this implies in turn that $u_{\alpha}$ is bounded in $L_{2^{*}}(M)$ and then in $L_{2}(M)$. Furthermore, we have

$$
\int_{M}\left|\nabla u_{\alpha}\right|^{2} d v_{g}=n J_{\alpha}\left(u_{\alpha}\right)+\int \frac{h_{\alpha}}{\rho_{p}^{2}} u_{\alpha}^{2} d v_{g}+o\left(\|u\|_{H_{1}^{2}(M)}\right) .
$$

By continuity of $h_{\alpha}$ on $p$, we have that for all $\epsilon>0$ there exists $\delta>0$ such that

$$
\int_{M}\left|\nabla u_{\alpha}\right|^{2} d v_{g} \leq n \beta+\left(\varepsilon+h_{\alpha}(p)\right) \int_{B(p, \delta)} \frac{u_{\alpha}^{2}}{\rho_{p}^{2}} d v_{g}+\delta^{-2} \int_{M \backslash B(p, \delta)} h_{\alpha} u_{\alpha}^{2} d v_{g}+o\left(\|u\|_{H_{1}^{2}(M)}\right)+o(1),
$$

then, by applying Hardy inequality (2.3) that for every $\varepsilon>0$ small there exists a constant $A(\varepsilon)$ such that

$$
\begin{aligned}
\int_{M}\left|\nabla u_{\alpha}\right|^{2} d v_{g} \leq & n \beta+\left(\varepsilon+h_{\alpha}(p)\right)\left(\varepsilon+K^{2}(n, 2,-2)\right) \int_{M}\left|\nabla u_{\alpha}\right|^{2} d v_{g} \\
& +A(\varepsilon) \int_{M} u_{\alpha}^{2} d v_{g}+o\left(\|u\|_{H_{1}^{2}(M)}\right)+o(1)
\end{aligned}
$$

since $0<h_{\alpha}(p)<\frac{1}{K^{2}(n, 2,-2)}$, we can find $\varepsilon>0$ small such that $1-\left(\varepsilon+h_{\alpha}(p)\right)\left(\varepsilon+K^{2}(n, 2,-2)\right)>$ 0 , which implies that $\int_{M}\left|\nabla u_{\alpha}\right|^{2} d v_{g}$ is bounded. Thus, $u_{\alpha}$ bounded in $H_{1}^{2}(M)$.

Now, for two functions $\varphi, \phi \in H_{1}^{2}(M)$, Hölder and Hardy inequalities give

$$
\int_{M}\left|\frac{h_{\alpha}-h_{\infty}}{\rho_{p}^{2}} \phi \varphi\right| d v_{g} \leq C|| \varphi \|_{H_{1}^{2}(M)}|| \phi||_{H_{1}^{2}(M)} \sup _{M}\left|h_{\alpha}-h_{\infty}\right|
$$

writing

$$
\int_{M} \frac{h_{\alpha}}{\rho_{p}^{2}} \phi \varphi d v_{g}=\int_{M} \frac{h_{\alpha}-h_{\infty}}{\rho_{p}^{2}} \phi \varphi d v_{g}+\int_{M} \frac{h_{\infty}}{\rho_{p}^{2}} \phi \varphi d v_{g}
$$


we get by the assumption made on the sequence $h_{\alpha}$ that

$$
\int_{M} \frac{h_{\alpha}}{\rho_{p}^{2}} \phi \varphi d v_{g}=\int_{M} \frac{h_{\infty}}{\rho_{p}^{2}} \phi \varphi d v_{g}+o(1) .
$$

Then, since the sequence $u_{\alpha}$ is bounded in $H_{1}^{2}(M)$, by taking $\phi=u_{\alpha}$, we get from (3.1) together with the weak convergence of $u_{\alpha}$ to $u$ in $L^{2}\left(M, \rho^{-2}\right)$ that

$$
\int_{M} \frac{h_{\alpha}}{\rho_{p}^{2}} u_{\alpha} \varphi d v_{g}=\int_{M} \frac{h_{\infty}}{\rho_{p}^{2}} u \varphi d v_{g}+o(1)
$$

thus, applying the last identity to $\varphi=u$, we get by the weak convergence in $H_{1}^{2}(M)$ that

$$
J_{\alpha}\left(v_{\alpha}\right)=J_{\alpha}\left(u_{\alpha}\right)-J_{\infty}(u)+\Phi\left(u_{\alpha}\right)+o(1),
$$

with

$$
\Phi_{\alpha}\left(u_{\alpha}\right)=\frac{1}{2^{*}} \int_{M} f\left(\left|u_{\alpha}\right|^{2^{*}}-|u|^{2^{*}}-\left|v_{\alpha}\right|^{2^{*}}\right) d v_{g},
$$

which by the Brezis-Lieb convergence Lemma equals to $o(1)$, hence we obtain

$$
J_{\alpha}\left(v_{\alpha}\right)=\beta-J_{\infty}(u)+o(1) .
$$

Moreover, for $\varphi \in H_{1}^{2}(M)$, by taking $\phi=u$ in (3.2), we can write

$$
D J_{\alpha}\left(v_{\alpha}\right) \varphi=D J_{\alpha}\left(u_{\alpha}\right) \varphi-D J_{\infty}(u) \varphi+\Phi\left(v_{\alpha}\right) \varphi+o(1),
$$

with

$$
\Phi\left(v_{\alpha}\right) \varphi=\int_{M} f\left(\left|v_{\alpha}+u\right|^{2^{*}-2}\left(v_{\alpha}+u\right)-\left|v_{\alpha}\right|^{2^{*}-2} v_{\alpha}-|u|^{2^{*}-2} u\right) \varphi d v_{g} .
$$

Knowing that there exists a positive constant $C$ independent of $\alpha$ such that

$$
|| v_{\alpha}+\left.u\right|^{2^{*}-2}\left(v_{\alpha}+u\right)-\left|v_{\alpha}\right|^{2^{*}-2} v_{\alpha}-|u|^{2^{*}-2} u \mid \leq C\left(\left|v_{\alpha}\right|^{2^{*}-2}|u|+|u|^{2^{*}-2}\left|v_{\alpha}\right|\right),
$$

we get, after applying Hölder inequality, that there exists a positive constant $C$ such that

$$
\left|\Phi\left(v_{\alpha}\right) \varphi\right| \leq C\left(\left\|\left|v_{\alpha}\right|^{2^{*}-2}|u|\right\|_{L_{\frac{2^{*}}{2^{*}-1}}(M)}+\left\||u|^{2^{*}-2}\left|v_{\alpha}\right|\right\|_{L_{\frac{2^{*}}{2^{*}-1}}(M)}\right)\|\varphi\|_{L_{2^{*}}(M)},
$$

which gives that $\Phi\left(v_{\alpha}\right) \varphi=o(1), \forall \varphi \in H_{1}^{2}(M)$, since both $\frac{2^{*}\left(2^{*}-2\right)}{2^{*}-1}$ and $\frac{2^{*}}{2^{*}-1}$ are smaller than $2^{*}$ and the inclusion of $H_{1}^{2}(M)$ in $L_{q}(M)$ is compact for $q<2^{*}$.

On the other hand, since the sequence $u_{\alpha}^{2^{*}-2} u_{\alpha}$ is bounded in $L_{\frac{2^{*}}{2^{*}-1}}(M)$ and converges almost everywhere to $u^{2^{*}-2} u$, we get that $u_{\alpha}^{2^{*}-2} u_{\alpha}$ converges weakly in $L_{\frac{2^{*}}{2^{*}-1}}(M)$ to $u^{2^{*}-2} u$. This, together with the weak convergence in $H_{1}^{2}(M)$ of $u_{\alpha}$ to $u$ and relation (3.3), imply that $D J_{\infty}(u) \varphi=0, \forall \varphi \in H_{1}^{2}(M)$. Hence, $D J_{\alpha}\left(v_{\alpha}\right) \varphi \rightarrow 0, \forall \varphi \in H_{1}^{2}(M)$. 
Lemma 3.2. Let $v_{\alpha}$ be a Palais-Smale sequence of $J_{\alpha}$ at level $\beta$ that converges weakly to 0 in $H_{1}^{2}(M)$. If

$$
\beta<\beta^{*}=\frac{\left(1-h_{\infty}(p) K^{2}(n, 2,-2)\right)^{\frac{n}{2}}}{n\left(\sup _{M} f\right)^{\frac{n-2}{2}} K(n, 2)^{n}},
$$

then $v_{\alpha}$ converges strongly to 0 in $H_{1}^{2}(M)$.

Proof. If $v_{\alpha}$ is a Palais-Smale sequence of $J_{\alpha}$ at level $\beta$ that converges to 0 weakly in $H_{1}^{2}(M)$, then $\int_{M} v_{\alpha}^{2} d v_{g}=o(1)$ and

$$
\beta=\frac{1}{n} \int_{M}\left(\left|\nabla v_{\alpha}\right|^{2}-\frac{h_{\alpha}}{\rho_{p}^{2}} v_{\alpha}^{2}\right) d v_{g}=\frac{1}{n} \int_{M} f\left|v_{\alpha}\right|^{2^{*}} d v_{g}+o(1) .
$$

This implies that $\beta \geq 0$. Hence, on the one hand, by Hardy inequality (2.3) we get as in Lemma 3.1, that for small enough $\varepsilon>0$,

$$
\int_{M}\left|\nabla v_{\alpha}\right|^{2} d v_{g} \leq \frac{n \beta}{1-\left[\left(h_{\alpha}(p)+\varepsilon\right)\left(\varepsilon+K^{2}(n, 2,-2)\right)\right]}+o(1),
$$

and on the other hand, by Sobolev inequality (2.1), we also get

$$
\int_{M}\left|\nabla v_{\alpha}\right|^{2} d v_{g} \geq\left(\frac{n \beta}{\left(\sup _{M} f\right) K^{2^{*}}(n, 2)}\right)^{\frac{2}{2^{*}}}+o(1) .
$$

Now, suppose that $\beta>0$, then the above inequalities (3.4) and (3.5), for $\alpha$ big enough, give

$$
\beta \geq \frac{\left.\left(1-\left(h_{\infty}(p)+2 \varepsilon\right)\left(K^{2}(n, 2,-2)+\varepsilon\right)\right)\right)^{\frac{n}{2}}}{n\left(\sup _{M} f\right)^{\frac{n-2}{2}} K(n, 2)^{n}},
$$

that is

$$
\beta^{\frac{2}{n}} \geq \beta^{* \frac{2}{n}}-\frac{2 \varepsilon^{2}+\varepsilon\left(h_{\infty}(p)+2 \varepsilon K^{2}(n, 2,-2)\right)}{n^{\frac{2}{n}}\left(\sup _{M} f\right)^{\frac{n-2}{n}} K(n, 2)^{2}} .
$$

By assumption $\beta^{*}>\beta$, by taking $\varepsilon>0$ small enough so that

$$
-2 \varepsilon^{2}-\varepsilon\left(h_{\infty}(p)-2 \varepsilon K^{2}(n, 2,-2)\right)+n^{\frac{2}{n}}\left(\sup _{M} f\right)^{\frac{n-2}{n}} K(n, 2)^{2}\left(\beta^{* \frac{2}{n}}-\beta^{\frac{2}{n}}\right)>0,
$$

we get a contradiction. Thus $\beta=0$ and (3.4) assures that

$$
\int_{M}\left|\nabla v_{\alpha}\right|^{2} d v_{g}=o(1)
$$

that is $v_{\alpha} \rightarrow 0$ strongly in $H_{1}^{2}(M)$.

In the following, for a given positive constant $R$, define a cut-off function $\eta_{R} \in C_{o}^{\infty}\left(I R^{n}\right)$ such that $\eta_{R}(x)=1, x \in B(R)$ and $\eta_{R}(x)=0, x \in I R^{n} \backslash B(2 R), 0 \leq \eta_{R} \leq 1$ and $\left|\nabla \eta_{R}\right| \leq \frac{C}{R}$. 
Lemma 3.3. Let $v_{\alpha}$ be Palais-Smale sequence for $J_{\alpha}$ at level $\beta$ that weakly, but not strongly, converges to 0 in $H_{1}^{2}(M)$. Then, there exists a sequence of positive reals $R_{\alpha} \rightarrow 0$ such that, up to a subsequence, $\hat{\eta}_{\alpha} \hat{v}_{\alpha}$ with

$$
\hat{v}_{\alpha}(x)=R_{\alpha}^{\frac{n-2}{2}} v_{\alpha}\left(\exp _{p}\left(R_{\alpha} x\right)\right),
$$

and $\hat{\eta}_{\alpha}(x)=\eta_{\delta}\left(R_{\alpha} x\right)$ ) ( $\delta$ is some positive constant), converges weakly in $D^{1,2}\left(\mathbb{R}^{n}\right)$ to a function $v \in D_{1}^{2}\left(\mathbb{R}^{n}\right)$ such that, if $v \neq 0, v$ is a weak solution of the Euclidean equation

$$
\Delta v-\frac{h_{\infty}(p)}{|x|^{2}} v=f(p)|v|^{2^{*}-2} v
$$

Proof. Since the Palais-Smale sequence $v_{\alpha}$ of $J_{\alpha}$ at level $\beta$ converges weakly and not strongly in $H_{1}^{2}(M)$ to 0 , we get by Lemma 3.2 that $\beta \geq \beta^{*}$. Write

$$
\int_{M}\left(\left|\nabla v_{\alpha}\right|^{2}-\frac{h_{\alpha}}{\rho_{p}^{2}} v_{\alpha}^{2}\right) d v_{g}=\int_{M} f\left|v_{\alpha}\right|^{2^{*}} d v_{g}+o(1)=n \beta+o(1),
$$

since, up to a subsequence, $v_{\alpha}$ converges strongly to 0 in $L_{2}(M)$, we get by Hardy inequality (2.3) that for all $\varepsilon>0$ small

$$
n \beta^{*}+o(1) \leq \int_{M}\left|\nabla v_{\alpha}\right|^{2} d v_{g} \leq \frac{n \beta}{1-\left(h_{\alpha}(p)+\varepsilon\right)\left(K^{2}(n, 2,-2)+\varepsilon\right)}+o(1) .
$$

In other words,

$$
c_{1} \leq \int_{M}\left|\nabla v_{\alpha}\right|^{2} d v_{g} \leq c_{2}
$$

for some positive constants $c_{1}$ and $c_{2}$.

Let $\gamma$ a small positive constant such that

$$
\limsup _{\alpha \rightarrow \infty} \int_{M}\left|\nabla v_{\alpha}\right|^{2}>\gamma
$$

Up to a subsequence, for each $\alpha>0$, we can find the smallest constant $r_{\alpha}>0$ such that

$$
\int_{B\left(p, r_{\alpha}\right)}\left|\nabla v_{\alpha}\right|^{2} d v_{g}=\gamma
$$

For a sequence of positive constants $R_{\alpha}$ and $x \in B\left(R_{\alpha}^{-1} \delta_{g}\right) \subset \mathbb{R}^{n}$, define

$$
\begin{aligned}
& \hat{v}_{\alpha}(x)=R_{\alpha}^{\frac{n-2}{2}} v_{\alpha}\left(\exp _{p}\left(R_{\alpha} x\right)\right), \\
& \left.\hat{g}_{\alpha}(x)=\left(\exp _{p}^{*} g\right)\left(R_{\alpha} x\right)\right) .
\end{aligned}
$$

We follow the same arguments as in [2]. Let $r>0$ be a constant and $z \in \mathbb{R}^{n}$ be such that $|z|+r<\delta_{g} R_{\alpha}^{-1}$, then we have

$$
\int_{B(z, r)}\left|\nabla \hat{v}_{\alpha}\right|^{2} d v_{\hat{g}}=\int_{\exp _{p}\left(R_{\alpha} B(z, r)\right)}\left|\nabla v_{\alpha}\right|^{2} d v_{g} .
$$


Let $0<r_{o}<\frac{\delta_{g}}{2}$ be such that for any $x, y \in B\left(r_{o}\right) \subset \mathbb{R}^{n}$, the following inequality holds

$$
\operatorname{dist}_{g}\left(\exp _{p}(x), \exp _{p}(y)\right) \leq C_{o}|x-y|
$$

for some positive constant $C_{o}$. Also, for $r \in\left(0, r_{0}\right)$, take $R_{\alpha}$ be such that $c_{o} r R_{\alpha}=r_{\alpha}$, then we get

$$
\left.\exp _{p}\left(R_{\alpha} B\left(C_{o} r\right)\right)\right)=B\left(p, C_{o} r R_{\alpha}\right)
$$

and then

$$
\int_{B\left(C_{o} r\right)}\left|\nabla \hat{v}_{\alpha}\right|^{2} d v_{\hat{g}}=\int_{B\left(p, r_{\alpha}\right)}\left|\nabla v_{\alpha}\right|^{2} d v_{g}=\gamma
$$

Take $\delta$ such that $0<\delta \leq \min \left(C_{o} r, \frac{\delta_{g}}{2}\right)$, there exists a positive constant such that, for all $u \in D^{1,2}\left(\mathbb{R}^{n}\right)$ with $\operatorname{Supp}(u) \in B\left(\delta R_{\alpha}^{-1}\right)$, the following inequalities hold

$$
\begin{aligned}
& \frac{1}{C_{1}} \int_{\mathbb{R}^{n}}|\nabla u|^{2} d x \leq \int_{\mathbb{R}^{n}}|\nabla u|^{2} d v_{\hat{g}} \leq C_{1} \int_{\mathbb{R}^{n}}|\nabla u|^{2} d x, \\
& \frac{1}{C_{1}} \int_{\mathbb{R}^{n}}|u| d x \leq \int_{\mathbb{R}^{n}}|u| d v_{\hat{g}} \leq C_{1} \int_{\mathbb{R}^{n}}|u| d x .
\end{aligned}
$$

Define a sequence of cut-off functions $\hat{\eta}_{\alpha}$ by $\hat{\eta}_{\alpha}(x)=\eta_{\delta}\left(R_{\alpha} x\right)$. Then, it follows from (3.10), (3.11a) and (3.11b) that the sequence $\tilde{v}_{\alpha}=\hat{\eta}_{\alpha} \hat{v}_{\alpha}$ is bounded in $D^{1,2}\left(I R^{n}\right)$. Consequently, up to a subsequence, $\tilde{v}_{\alpha}$ converges weakly to some function $v \in D^{1,2}\left(I^{n}\right)$.

Suppose that $v \neq 0$, since $v_{\alpha}$ converges weakly to 0 , it follows that $R_{\alpha} \rightarrow 0$.

Let us first prove that $v$ is a weak solution on $D^{1,2}\left(I R^{n}\right)$ to (3.6). For this task, we let $\varphi \in \mathcal{C}_{o}^{\infty}\left(\mathbb{R}^{n}\right)$ be a function with compact support included in the ball $B(\delta)$. For $\alpha$ large, define on $M$ the sequence $\varphi_{\alpha}$ as

$$
\varphi_{\alpha}(x)=R_{\alpha}^{\frac{2-n}{2}} \varphi\left(R_{\alpha}^{-1}\left(\exp _{p}^{-1}(x)\right)\right) .
$$

Then, we have

$$
\begin{aligned}
& \int_{M} \nabla v_{\alpha} \nabla \varphi_{\alpha} d v_{g}=\int_{\mathbb{R}^{n}} \nabla \tilde{v}_{\alpha} \nabla \varphi d v_{\hat{g}_{\alpha}} \\
& \int_{M} \frac{h_{\alpha}}{\rho_{p}^{2}} v_{\alpha} \varphi_{\alpha} d v_{g}=R_{\alpha}^{2} \int_{\mathbb{R}^{n}} \frac{h_{\alpha}\left(\exp _{p}\left(R_{\alpha} x\right)\right)}{d i s t_{\hat{g}_{\alpha}}^{2}\left(0, R_{\alpha} x\right)} \tilde{v}_{\alpha} \varphi d v_{\hat{g}_{\alpha}} \\
& \int_{M} f\left|v_{\alpha}\right|^{2^{*}-2} v_{\alpha} \varphi_{\alpha} d v_{g} \int_{\mathbb{R}^{n}} f\left(\exp _{p}\left(R_{\alpha} x\right)\right)\left|\tilde{v}_{\alpha}\right|^{2^{*}-2} \tilde{v}_{\alpha} \varphi d v_{\hat{g}_{\alpha}} .
\end{aligned}
$$

When tending $\alpha$ to $\infty, \hat{g}_{\alpha}$ tends smoothly to the Euclidean metric on $I R^{n}$, then by passing to the limit when $\alpha \rightarrow \infty$ and since $v_{\alpha}$ is a Palais-Smale sequence of $J_{\alpha}$, we get that $v$ is weak solution of (3.6). 
Lemma 3.4. Let $v$ be the solution of (3.6) given by Lemma 3.3, then up to a subsequence,

$$
w_{\alpha}=v_{\alpha}-R_{\alpha}^{\frac{2-n}{2}} \eta_{\delta}\left(\exp ^{-1}(x)\right) v\left(R_{\alpha}^{-1} \exp _{p}^{-1}(x)\right),
$$

where $0<\delta<\frac{\delta_{g}}{2}$, is a Palais-Sequence for $J_{\alpha}$ at level $\beta-G_{\infty}(v)$ that weakly converges to 0 in $H_{1}^{2}(M)$.

Proof. For $0<\delta<\frac{\delta_{g}}{2}$, define

$$
\mathcal{B}_{\alpha}(x)=R_{\alpha}^{\frac{2-n}{2}} \eta_{\delta}\left(\exp _{p}^{-1}(x)\right) v\left(R_{\alpha}^{-1} \exp _{p}^{-1}(x)\right), \quad x \in M,
$$

and put

$$
w_{\alpha}=v_{\alpha}-\mathcal{B}_{\alpha} .
$$

We begin proving that $w_{\alpha}$ converges weakly to 0 in $H_{1}^{2}(M)$, it suffices to prove that $\mathcal{B}_{\alpha}$ does. Take a function $\varphi \in \mathrm{C}^{\infty}(M)$, then we have

$$
\begin{aligned}
& \int_{B(p, 2 \delta)}\left(\nabla \mathcal{B}_{\alpha} \nabla \varphi+\mathcal{B}_{\alpha} \varphi\right) d v_{g} \\
= & R_{\alpha}^{\frac{n}{2}} \int_{B\left(2 \delta R_{\alpha}^{-1}\right)}\left[R_{\alpha} v(x)\left(\nabla \eta_{\delta}\right)\left(R_{\alpha} x\right)+\eta_{\delta}\left(R_{\alpha} x\right) \nabla v\right] \nabla \varphi\left(\exp _{p}\left(R_{\alpha} x\right)\right) d v_{\hat{g}_{\alpha}} \\
& +R_{\alpha}^{\frac{n+2}{2}} \int_{B\left(2 \delta R_{\alpha}^{-1}\right)} v \eta_{\delta}\left(R_{\alpha} x\right) \varphi\left(\exp _{p}\left(R_{\alpha} x\right)\right) d v_{\hat{g}_{\alpha}},
\end{aligned}
$$

then, for a positive constant $C^{\prime}$ such that $d v_{\hat{g}_{\alpha}} \leq C^{\prime} d x$, it follows that

$$
\begin{aligned}
& \int_{B(p, 2 \delta)}\left(\nabla \mathcal{B}_{\alpha} \nabla \varphi+\mathcal{B}_{\alpha} \varphi\right) d v_{g} \\
\leq & \left.C^{\prime} R_{\alpha}^{\frac{n}{2}}\left[\sup _{M}|\nabla \varphi| \int_{I R^{n}}\left(|\nabla v|+|v| C \delta^{-1}\right) d x+R_{\alpha} \sup _{M}|\varphi| \int_{I R^{n}}|v|\right) d x\right] .
\end{aligned}
$$

Thus, when tending $\alpha \rightarrow \infty$, we ge that $\mathcal{B}_{\alpha} \rightarrow 0$ weakly in $H_{1}^{2}(M)$.

Now, let us evaluate $J_{\alpha}\left(w_{\alpha}\right)$. First, we have

$$
\int_{M}\left|\nabla w_{\alpha}\right|^{2} d v_{g}=\int_{M \backslash B(p, 2 \delta)}\left|\nabla v_{\alpha}\right|^{2} d v_{g}+\int_{B(p, 2 \delta)}\left|\nabla\left(v_{\alpha}-\mathcal{B}_{\alpha}\right)\right|^{2} d v_{g}
$$

and of course

$$
\begin{aligned}
& \int_{B(p, 2 \delta)}\left|\nabla\left(v_{\alpha}-\mathcal{B}_{\alpha}\right)\right|^{2} d v_{g} \\
= & \int_{B(p, 2 \delta)}\left|\nabla v_{\alpha}\right|^{2} d v_{g}-2 \int_{B(p, 2 \delta)} \nabla v_{\alpha} \nabla \mathcal{B}_{\alpha} d v_{g}+\int_{B(p, 2 \delta)}\left|\nabla \mathcal{B}_{\alpha}\right|^{2} d v_{g} .
\end{aligned}
$$


Direct calculation gives

$$
\begin{aligned}
\int_{B(p, 2 \delta)}\left|\nabla \mathcal{B}_{\alpha}\right|^{2} d v_{g}= & \int_{B\left(2 \delta R_{\alpha}^{-1}\right)} \eta_{\delta}^{2}\left(R_{\alpha} x\right)|\nabla v|^{2} d v_{\hat{g}_{\alpha}} \\
& +R_{\alpha}^{2} \int_{B\left(2 \delta R_{\alpha}^{-1}\right)} v^{2}\left|\nabla \eta_{\delta}\right|^{2}\left(R_{\alpha} x\right) d v_{\hat{g}_{\alpha}}+2 R_{\alpha} \nabla \eta_{\delta}\left(R_{\alpha} x\right) \nabla v d v_{\hat{g}_{\alpha}} .
\end{aligned}
$$

It can be easily seen that the second term of right-hand side member of the above equality tends to 0 as $\alpha \rightarrow \infty$. Furthermore, for $R>0$, a positive constant, we write

$$
\int_{B\left(2 \delta R_{\alpha}^{-1}\right)} \eta_{\delta}^{2}\left(R_{\alpha} x\right)|\nabla v|^{2} d v_{\hat{g}_{\alpha}}=\int_{B(R)} \eta_{\delta}^{2}\left(R_{\alpha} x\right)|\nabla v|^{2} d v_{\hat{g}_{\alpha}}+\int_{\mathbb{R}^{n} \backslash B(R)} \eta_{\delta}^{2}\left(R_{\alpha} x\right)|\nabla v|^{2} d v_{\hat{g}_{\alpha}}
$$

with

$$
\int_{\mathbb{R}^{n} \backslash B(R)} \eta_{\delta}^{2}\left(R_{\alpha} x\right)|\nabla v|^{2} d v_{\hat{g}_{\alpha}} \leq C \int_{\mathbb{R}^{n} \backslash B(R)}|\nabla v|^{2} d x=\varepsilon_{R},
$$

where $\varepsilon_{R}$ is a function in $R$ such that $\varepsilon_{R} \rightarrow 0$ as $R \rightarrow \infty$.

Noting that $\hat{g}_{\alpha}$ goes locally in $C^{1}$ to the Euclidean metric $\xi$, we get then

$$
\int_{B(p, 2 \delta)}\left|\nabla \mathcal{B}_{\alpha}\right|^{2} d v_{g}=\int_{\mathbb{R}^{n}}|\nabla v|^{2} d x+o(1)+\varepsilon_{R}
$$

Moreover, we have

$$
\begin{aligned}
& \int_{B(p, 2 \delta)} \nabla v_{\alpha} \nabla \mathcal{B}_{\alpha} d v_{g} \\
= & \int_{B\left(2 \delta R_{\alpha}^{-1}\right)} \nabla\left(\eta_{\delta}\left(R_{\alpha} x\right) \hat{v}_{\alpha}\right) \nabla v d v_{\hat{g}_{\alpha}}+R_{\alpha} \int_{B\left(2 \delta R_{\alpha}^{-1}\right)}\left(v \nabla \hat{v}_{\alpha}-\hat{v}_{\alpha} \nabla v\right) \nabla \eta_{\delta}\left(R_{\alpha} x\right) d v_{\hat{g}_{\alpha}}
\end{aligned}
$$

with

$$
\begin{aligned}
& \left|\int_{B\left(2 \delta R_{\alpha}^{-1}\right)} \nabla \eta_{\delta}\left(R_{\alpha} x\right)\left(v \nabla \hat{v}_{\alpha}-\hat{v}_{\alpha} \nabla v\right) d v_{\hat{g}_{\alpha}}\right| \\
& \leq c \delta^{-1}\left[\left(\int_{B\left(2 \delta R_{\alpha}^{-1}\right)}\left|\nabla \hat{v}_{\alpha}\right|^{2} d v_{\hat{g}_{\alpha}}\right)^{\frac{1}{2}}\left(\int_{B\left(2 \delta R_{\alpha}^{-1}\right)} v^{2} d x\right)^{\frac{1}{2}}\right. \\
& \left.\quad+\left(\int_{B\left(2 \delta R_{\alpha}^{-1}\right)} \hat{v}_{\alpha}^{2} d v_{\hat{g}_{\alpha}}\right)^{\frac{1}{2}}\left(\int_{\left.B\left(2 \delta R_{\alpha}^{-1}\right)\right)}|\nabla v|^{2} d x\right)^{\frac{1}{2}}\right] .
\end{aligned}
$$

Since $v_{\alpha}$ is bounded in $H_{1}^{2}(M)$, the quantities $\int_{B\left(2 \delta R_{\alpha}^{-1}\right)}\left|\nabla \hat{v}_{\alpha}\right|^{2} d v_{\hat{g}_{\alpha}}$ and $\int_{B\left(2 \delta R_{\alpha}^{-1}\right)}\left|\hat{v}_{\alpha}\right|^{2} d v_{\hat{g}_{\alpha}}$ are bounded and hence the second term of the right-hand side member of (3.13) is $o(1)$. Thus, by using the weak convergence of $\hat{\eta}_{\alpha} \hat{v}_{\alpha}$ to $v$ in $D^{1,2}\left(I R^{n}\right)$ that

$$
\int_{B(p, \delta)} \nabla v_{\alpha} \nabla \mathcal{B}_{\alpha} d v_{g}=\int_{\mathbb{R}^{n}}|\nabla v|^{2} d x+o(1),
$$


so that

$$
\int_{M}\left|\nabla w_{\alpha}\right|^{2} d v_{g}=\int_{M}\left|\nabla v_{\alpha}\right|^{2} d v_{g}-\int_{\mathbb{R}^{n}}|\nabla v|^{2} d x+o(1)+\varepsilon_{R} .
$$

In the same fashion, for $R$ a positive constant and $\alpha$ large, we write

$$
\int_{B(p, 2 \delta)} \frac{h_{\alpha}}{\rho_{p}^{2}} \mathcal{B}_{\alpha}^{2} d v_{g}=\int_{B\left(p, R R_{\alpha}\right)} \frac{h_{\alpha}}{\rho_{p}^{2}} \mathcal{B}_{\alpha}^{2} d v_{g}+\int_{B(p, 2 \delta) \backslash B\left(p, R R_{\alpha}\right)} \frac{h_{\alpha}}{\rho_{p}^{2}} \mathcal{B}_{\alpha}^{2} d v_{g}
$$

with

$$
\int_{B(p, 2 \delta) \backslash B\left(p, R R_{\alpha}\right)} \frac{h_{\alpha}}{\rho_{p}^{2}} \mathcal{B}_{\alpha}^{2} d v_{g} \leq C\left(R R_{\alpha}\right)^{-2} \int_{B(p, 2 \delta) \backslash B\left(p, R R_{\alpha}\right)} \mathcal{B}_{\alpha}^{2} d v_{g},
$$

then, by a direct calculations, we get

$$
\int_{B(p, 2 \delta) \backslash B\left(p, R R_{\alpha}\right)} \frac{h_{\alpha}}{\rho_{p}^{2}} \mathcal{B}_{\alpha}^{2} d v_{g} \leq C R^{-2} \int_{I R^{n} \backslash B(R)} v^{2} d x=\varepsilon_{R} .
$$

Hence,

$$
\begin{aligned}
\int_{B(p, 2 \delta)} \frac{h_{\alpha}}{\rho_{p}^{2}} \mathcal{B}_{\alpha}^{2} & =R_{\alpha}^{2} \int_{B(R)} \frac{h_{\alpha}\left(\exp _{p}\left(R_{\alpha} x\right)\right)}{\left(\operatorname{dist}_{\hat{g}_{\alpha}}\left(0, R_{\alpha} x\right)^{2}\right.} \eta_{\alpha}^{2}\left(R_{\alpha} x\right) v^{2} d v_{\hat{g}_{\alpha}}+\varepsilon_{R} \\
& =h_{\infty}(p) \int_{\mathbb{R}^{n}} \frac{v^{2}}{|x|^{2}} d x+o(1)+\varepsilon_{R}
\end{aligned}
$$

Also, in similar way, since $v_{\alpha}$ is bounded in $H_{1}^{2}(M)$, after using Hölder and Hardy inequalities, we can easily have

$$
\int_{B(p, 2 \delta) \backslash B\left(p, R R_{\alpha}\right)} \frac{h_{\alpha}}{\rho_{p}^{2}} v_{\alpha} \mathcal{B}_{\alpha} d v_{g} \leq C R^{-2} \int_{I R^{n} \backslash B(R)} v^{2} d v_{g}=\varepsilon_{R},
$$

which yields

$$
\begin{aligned}
\int_{B(p, \delta)} \frac{h_{\alpha}}{\rho_{p}^{2}} v_{\alpha} \mathcal{B}_{\alpha} d v_{g} & =R_{\alpha}^{2} \int_{B(R)} \frac{h_{\alpha}\left(\exp _{p}\left(R_{\alpha} x\right)\right)}{\left(\operatorname{dist}_{\hat{g}_{\alpha}}\left(0, R_{\alpha} x\right)\right)^{2}}\left(\eta\left(R_{\alpha} x\right) \hat{v}_{\alpha}\right) v d v_{\hat{g}_{\alpha}}+\varepsilon_{R} \\
& =h_{\infty}(p) \int_{\mathbb{R}^{n}} \frac{v^{2}}{|x|^{2}} d x+o(1)+\varepsilon_{R} .
\end{aligned}
$$

so that in the end we obtain

$$
\int_{M} \frac{h_{\alpha}}{\rho_{p}^{2}} w_{\alpha}^{2} d v_{g}=\int_{M} \frac{h_{\alpha}}{\rho_{p}^{2}} v_{\alpha}^{2} d v_{g}-h_{\infty}(p) \int_{\mathbb{R}^{n}} \frac{v^{2}}{|x|^{2}} d x+o(1)+\varepsilon_{R}
$$

In similar way, we can prove that

$$
\int_{M}\left|w_{\alpha}\right|^{2^{*}} d v_{g}=\int_{M}\left|v_{\alpha}\right|^{2^{*}} d v_{g}-f(p) \int_{M}|v|^{2^{*}} d v_{g}+o(1)+\varepsilon_{R}
$$


Finally, since $R$ is arbitrary, when summing up we obtain

$$
J_{\alpha}\left(w_{\alpha}\right)=J_{\alpha}\left(u_{\alpha}\right)-G_{\infty}(v)+o(1)=\beta-G_{\infty}(v)+o(1) .
$$

It remains to prove that $D J_{\alpha}\left(\mathcal{B}_{\alpha}\right) \rightarrow 0$ in $H_{1}^{2}(M)^{\prime}$. Let $\varphi \in H_{1}^{2}(M)$, for $x \in B\left(\delta R_{\alpha}^{-1}\right)$ put $\varphi_{\alpha}(x)=R_{\alpha}^{\frac{n-2}{2}} \varphi\left(\exp _{p}\left(R_{\alpha} x\right)\right)$ and $\left.\bar{\varphi}_{\alpha}(x)=\eta_{\delta}\left(R_{\alpha} x\right)\right) \varphi_{\alpha}(x)$, then we have

$$
\int_{B(p, 2 \delta)} \nabla \mathcal{B}_{\alpha} \nabla \varphi d v_{g}=\int_{B\left(2 \delta R_{\alpha}^{-1}\right)} \nabla v \nabla \bar{\varphi}_{\alpha} d v_{\hat{g}_{\alpha}}+R_{\alpha} \int_{B\left(2 \delta R_{\alpha}^{-1}\right)} \nabla \eta_{\delta}\left(R_{\alpha} x\right)\left(v \nabla \varphi_{\alpha}-\varphi_{\alpha} \nabla v\right) d v_{\hat{g}_{\alpha}} .
$$

Knowing that

$$
\int_{B(p, 2 \delta)}|\nabla \varphi|^{2} d v_{g}=\int_{B\left(2 \delta R_{\alpha}^{-1}\right)}\left|\nabla \varphi_{\alpha}\right|^{2} d v_{\hat{g}_{\alpha}}
$$

we get that

$$
\int_{B\left(2 \delta R_{\alpha}^{-1}\right)}\left|\nabla \eta_{\delta}\left(R_{\alpha} x\right)\left(v \nabla \varphi_{\alpha}-\varphi_{\alpha} \nabla v\right)\right| d v_{\hat{g}_{\alpha}} \leq C|| \varphi \|_{H_{1}^{2}(M)}
$$

which gives that

$$
\int_{B(p, 2 \delta)} \nabla \mathcal{B}_{\alpha} \nabla \varphi d v_{g}=\int_{B\left(2 \delta R_{\alpha}^{-1}\right)} \nabla v \nabla \bar{\varphi}_{\alpha} d v_{\hat{g}_{\alpha}}+o\left(\|\varphi\|_{H_{1}^{2}(M)}\right) .
$$

Next, for $R>0$ write

$$
\int_{B\left(2 \delta R_{\alpha}^{-1}\right)} \nabla v \nabla \bar{\varphi}_{\alpha} d v_{\hat{g}_{\alpha}}=\int_{B(R)} \nabla v \nabla \bar{\varphi}_{\alpha} d v_{\hat{g}_{\alpha}}+\int_{B\left(2 \delta R_{\alpha}^{-1}\right) \backslash B(R)} \nabla v \nabla \bar{\varphi}_{\alpha} d v_{\hat{g}_{\alpha}},
$$

note that

$$
\begin{aligned}
\int_{B\left(2 \delta R_{\alpha}^{-1}\right) \backslash B(R)} \nabla v \nabla \bar{\varphi}_{\alpha} d v_{\hat{g}_{\alpha}} & \leq C\|\varphi\|_{H_{1}^{2}(M)}\left(\int_{B\left(2 \delta R_{\alpha}^{-1}\right) \backslash B(R)}|\nabla v|^{2} d x\right)^{\frac{1}{2}} \\
& =\mathcal{O}\left(\|\varphi\|_{H_{1}^{2}(M)}\right) \varepsilon(R),
\end{aligned}
$$

where $\varepsilon_{R} \rightarrow 0$ as $R \rightarrow \infty$. Since the sequence of metrics $\hat{g}_{\alpha}$ tends locally in $C^{1}$ when $\alpha \rightarrow \infty$ to the Euclidean metric, we obtain

$$
\int_{B(p, 2 \delta)} \nabla \mathcal{B}_{\alpha} \nabla \varphi d v_{g}=\int_{I R^{n}} \nabla v \nabla \bar{\varphi}_{\alpha} d x+o\left(\|\varphi\|_{H_{1}^{2}(M)}\right)+\mathcal{O}\left(\|\varphi\|_{H_{1}^{2}(M)}\right) \varepsilon(R) .
$$

Moreover, for a given $R>0$, we have for $\alpha$ large,

$$
\int_{B(p, 2 \delta)} \frac{h_{\alpha}}{\rho_{p}^{2}} \mathcal{B}_{\alpha} \varphi d v_{g}=\int_{B\left(p, R R_{\alpha}\right)} \frac{h_{\alpha}}{\rho_{p}^{2}} \mathcal{B}_{\alpha} \varphi d v_{g}+\int_{B(p, 2 \delta) \backslash B\left(p, R R_{\alpha}\right)} \frac{h_{\alpha}}{\rho_{p}^{2}} \mathcal{B}_{\alpha} \varphi d v_{g} .
$$

On the one hand, we have

$$
\int_{B(p, 2 \delta) \backslash B_{p}\left(R R_{\alpha}\right)} \frac{h_{\alpha}}{\rho_{p}^{2}} \mathcal{B}_{\alpha} \varphi d v_{g} \leq \frac{C}{\left(R R_{\alpha}\right)^{2}}\|\varphi\|_{H_{1}^{2}(M)} \int_{B(p, 2 \delta) \backslash B\left(p, R R_{\alpha}\right)} \mathcal{B}_{\alpha}^{2} d v_{g},
$$


and a straightforward computation shows that

$$
\int_{B(p, 2 \delta) \backslash B\left(p, R R_{\alpha}\right)}\left|\mathcal{B}_{\alpha}\right|^{2} d v_{g} \leq C R_{\alpha}^{2} \int_{B\left(2 \delta R_{\alpha}^{-1}\right) \backslash B(R)} v^{2} d x,
$$

which implies that

$$
\int_{B(p, 2 \delta) \backslash B\left(p, R R_{\alpha}\right)} \frac{h_{\alpha}}{\rho_{p}^{2}} \mathcal{B}_{\alpha} \varphi d v_{g}=\mathcal{O}\left(\|\varphi\|_{H_{1}^{2}(M)}\right) \varepsilon_{R}
$$

with $\varepsilon_{R} \rightarrow 0$ as $R \rightarrow \infty$.

On the other hand, we have

$$
\int_{B\left(p, R R_{\alpha}\right)} \frac{h_{\alpha}}{\rho_{p}^{2}} \mathcal{B}_{\alpha} \varphi d v_{g}=R_{\alpha}^{2} \int_{B(R)} \frac{h_{\alpha}\left(\exp _{p} R_{\alpha} x\right)}{\left(\operatorname{dist}_{\hat{g}_{\alpha}}\left(0, R_{\alpha} x\right)\right)^{2}} v \bar{\varphi} d v_{\hat{\mathrm{g}}},
$$

which leads to

$$
\begin{aligned}
\int_{B_{p}\left(R R_{\alpha}\right)} \frac{h_{\alpha}}{\rho_{p}^{2}} \mathcal{B}_{\alpha} \varphi d v_{g} & =\int_{B(R)} \frac{h_{\infty}(p)}{|x|^{2}} v \bar{\varphi} d x+o\left(\|\varphi\|_{H_{1}^{2}(M)}\right) \\
& =\int_{\mathbb{R}^{n}} \frac{h_{\infty}(p)}{|x|^{2}} v \bar{\varphi} d x-\int_{\mathbb{R}^{n} \backslash B(R)} \frac{h_{\infty}(p)}{|x|^{2}} v \bar{\varphi} d x+o\left(\|\varphi\|_{H_{1}^{2}(M)}\right),
\end{aligned}
$$

with

$$
\int_{\mathbb{R}^{n} \backslash B(R)} \frac{h_{\infty}(p)}{|x|^{2}} v \bar{\varphi} d x \leq \frac{C}{R^{2}}\|\varphi\|_{H_{1}^{2}(M)}=\mathcal{O}\left(\|\varphi\|_{H_{1}^{2}(M)}\right) \varepsilon_{R}
$$

so that

$$
\int_{B(p, 2 \delta)} \frac{h_{\alpha}}{\rho_{p}^{2}} \mathcal{B}_{\alpha} \varphi d v_{g}=\int_{\mathbb{R}^{n}} \frac{h_{\infty}(p)}{|x|^{2}} v \bar{\varphi} d x+o\left(\|\varphi\|_{H_{1}^{2}(M)}\right)+\mathcal{O}\left(\|\varphi\|_{H_{1}^{2}(M)}\right) \varepsilon_{R} .
$$

In the same way, we can also have

$$
\int_{B(p, 2 \delta)} f\left|\mathcal{B}_{\alpha}\right|^{\frac{4}{n-2}} \mathcal{B}_{\alpha} \varphi d v_{g}=f(p) \int_{\mathbb{R}^{n}}|v|^{\frac{4}{n-2}} v \bar{\varphi}_{\alpha} d x+o\left(\|\varphi\|_{H_{1}^{2}(M)}\right)+\mathcal{O}\left(\|\varphi\|_{H_{1}^{2}(M)}\right) \varepsilon_{R} .
$$

Summing up, we obtain

$$
\begin{aligned}
& \int_{B(p, 2 \delta)}\left(\nabla \mathcal{B}_{\alpha} \nabla \varphi d v_{g}+\frac{h_{\alpha}}{\rho_{p}^{2}} \mathcal{B}_{\alpha} \varphi\right) d v_{g}-\int_{B(p, 2 \delta)} f\left|\mathcal{B}_{\alpha}\right|^{\frac{4}{n-2}} \mathcal{B}_{\alpha} \varphi d v_{g} \\
= & \int_{I R^{n}}\left(\nabla v \nabla \bar{\varphi}_{\alpha} d x+\frac{h_{\infty}(p)}{|x|^{2}} v \bar{\varphi}_{\alpha}\right) d x-f(p) \int_{\mathbb{R}^{n}}|v|^{\frac{4}{n-2}} v \bar{\varphi}_{\alpha} d x \\
& +o\left(\|\varphi\|_{H_{1}^{2}(M)}\right)+\mathcal{O}\left(\|\varphi\|_{H_{1}^{2}(M)}\right) \varepsilon_{R},
\end{aligned}
$$

and since $v$ is weak solution of $\left(E_{\infty}\right)$, we get the desired result. 
Keeping the notations adapted above, we prove the following lemma

Lemma 3.5. Let $v_{\alpha}$ a Palais-Smale sequence for $J_{\alpha}$ at level $\beta$. Suppose that the sequence $\tilde{v}=\hat{\eta}_{\alpha} \hat{v}_{\alpha}$ of the above lemma converges weakly to 0 in $D^{1,2}\left(I R^{n}\right)$. Then, there exist a sequence of positive numbers $\left\{\tau_{\alpha}\right\}, \tau_{\alpha} \rightarrow 0$ and a sequence of points $x_{i} \in M, x_{i} \rightarrow x_{o} \in M \backslash\{p\}$ such that up to $a$ subsequence, the sequence $\eta_{\delta}\left(\tau_{\alpha} x\right) v_{\alpha}$, with $\delta$ is some constant and

$$
v_{\alpha}=\tau_{\alpha}^{\frac{n-2}{2}} v_{\alpha}\left(\exp _{x_{i}}\left(\tau_{\alpha} x\right)\right)
$$

converges weakly to a nontrivial weak solution $v$ of the Euclidean equation

$$
\Delta v=f\left(x_{0}\right)|v|^{\frac{4}{n-2}} v
$$

and the sequence

$$
\mathcal{W}_{\alpha}=v_{\alpha}-\tau_{\alpha}^{\frac{2-n}{2}} \eta_{\delta}\left(\exp _{x_{i}}^{-1}(x)\right) v\left(\tau_{\alpha}^{-1} \exp _{x_{i}}^{-1}(x)\right)
$$

is a Palais-Smale sequence for $J_{\alpha}$ at level $\beta-f\left(x_{o}\right)^{\frac{4}{n-2}} G(v)$ that converges weakly to 0 in $H_{2}^{1}(M)$.

Proof. Suppose that the sequence $\tilde{v}_{\alpha}=\hat{\eta}_{\alpha} \hat{v}_{\alpha}$ converges weakly to 0 in $\mathcal{D}^{1,2}\left(I^{n}\right)$. Take a function $\varphi \in \mathcal{C}_{o}^{\infty}\left(B\left(C_{o} r\right)\right)$ and put $\varphi_{\alpha}(x)=\varphi\left(R_{\alpha}^{-1} \exp _{p}^{-1}(x)\right)$. As in [6] and [1], by the strong convergence of $\tilde{v}_{\alpha}$ to 0 in $L_{l o c}^{2}\left(I R^{n}\right)$, we have for $\alpha$ large

$$
\begin{aligned}
& \int_{I R^{n}}\left|\nabla\left(\tilde{v}_{\alpha} \varphi\right)\right|^{2} d v_{\hat{g}_{\alpha}}=\int_{I R^{n}} \nabla \tilde{v}_{\alpha} \nabla\left(\tilde{v}_{\alpha} \varphi^{2}\right) d v_{\hat{g}_{\alpha}}+o(1) \\
= & \int_{M} \nabla v_{\alpha} \nabla\left(v_{\alpha} \varphi_{\alpha}^{2}\right) d v_{g}+o(1) \\
= & \left\|\mathcal{D} J_{\alpha}\right\|\left\|v_{\alpha} \varphi_{\alpha}^{2}\right\|+\int_{M} \frac{h_{\alpha}}{\rho_{p}^{2}}\left(v_{\alpha} \varphi_{\alpha}\right)^{2} d v_{g}+\int_{M} f\left|v_{\alpha}\right|^{\frac{4}{n-2}}\left(v_{\alpha} \varphi_{\alpha}\right)^{2} d v_{g}+o(1) \\
\leq & \left(h_{\alpha}(p)+\varepsilon\right)\left(K^{2}(n, 2,-2)+\varepsilon\right) \int_{I R^{n}}\left|\nabla\left(\tilde{v}_{\alpha} \varphi\right)\right|^{2} d v_{\hat{g}_{\alpha}} \\
& \quad \sup _{M} f K^{2^{*}}(n, 2)\left(\int_{B\left(C_{o} r\right)}\left|\nabla \tilde{v}_{\alpha}\right|^{2} d v_{\hat{g}_{\alpha}}\right)^{\frac{2}{n-2}} \int_{I^{n}}\left|\nabla\left(\tilde{v}_{\alpha} \varphi\right)\right|^{2} d v_{\hat{g}_{\alpha}}+o(1) .
\end{aligned}
$$

Thus, for $\gamma$ chosen small enough, we get that for each $t, 0<t<C_{o r}$,

$$
\int_{B\left(p, t R_{\alpha}\right)}\left|\nabla v_{\alpha}\right|^{2} d v_{g}=\int_{B(t)}\left|\nabla \tilde{v}_{\alpha}\right|^{2} d v_{\hat{g}} \rightarrow 0 \quad \text { as } \quad \alpha \rightarrow \infty .
$$

Now, for $t>0$ consider the function

$$
t \longrightarrow \mathcal{F}(t)=\max _{x \in M} \int_{B(x, t)}\left|\nabla v_{\alpha}\right|^{2} d v_{g} .
$$

Since $\mathcal{F}$ is continuous, under (3.7) and (3.8), it follows that for any $\lambda \in(0, \gamma)$, there exist $t_{\alpha}>0$ small and $x_{\alpha} \in M$ such that

$$
\int_{B\left(x_{\alpha}, t_{\alpha}\right)}\left|\nabla v_{\alpha}\right|^{2} d v_{g}=\lambda
$$


Since $M$ is compact, up to a subsequence, we may assume that $x_{\alpha}$ converges to some point $x_{o} \in M$.

Note first that for all $\alpha \geq 0, t_{\alpha}<r_{\alpha}=C_{o} r R_{\alpha}$, otherwise if there exists $\alpha_{0} \geq 0$ such that $t_{\alpha_{0}}<r_{\alpha_{0}}$, we get a contradiction due to the fact that

$$
\lambda=\int_{B\left(x_{\alpha_{0}}, t_{\alpha_{0}}\right)}\left|\nabla v_{\alpha_{o}}\right|^{2} d v_{g} \geq \int_{B\left(p, t_{\alpha_{0}}\right)}\left|\nabla v_{\alpha_{o}}\right|^{2} d v_{g} \geq \int_{B\left(p, r_{\alpha_{0}}\right)}\left|\nabla v_{\alpha_{o}}\right|^{2} d v_{g}=\gamma
$$

Now, suppose that for all $\varepsilon>0$, there exists $\alpha_{\varepsilon}>0$ such that dist $t_{g}\left(x_{\alpha}, p\right) \leq \varepsilon$ for all $\alpha \geq \alpha_{\varepsilon}$. Choose $r_{\alpha}^{\prime}$ such that, $t_{\alpha}<r_{\alpha}^{\prime}<r_{\alpha}$ and take $\varepsilon^{\prime}=r_{\alpha}^{\prime}-t_{\alpha}$, we get that for some $\alpha_{\varepsilon^{\prime}}>0$ and $\alpha \geq \alpha_{\varepsilon^{\prime}}$

$$
B\left(x_{\alpha}, t_{\alpha}\right) \subset B\left(p, r_{\alpha}^{\prime}\right),
$$

which, by virtue of (3.16), is impossible. We deduce then that $x_{0} \neq p$.

Now, let $0<\tau_{\alpha}<1$, for $x \in B\left(\tau_{\alpha}^{-1} \delta_{g}\right) \subset \mathbb{R}^{n}$ consider the sequences

$$
\begin{aligned}
& v_{\alpha}(x)=\tau_{\alpha}^{\frac{n-2}{2}} v_{\alpha}\left(\exp _{x_{\alpha}}\left(\tau_{\alpha} x\right)\right), \\
& \left.\tilde{g}_{\alpha}(x)=\exp _{x_{\alpha}}^{*} g\left(\tau_{\alpha} x\right)\right) .
\end{aligned}
$$

Take $\tau_{\alpha}$ such that $C_{0} r \tau_{\alpha}=t_{\alpha}$. As in the above lemma, we can easily check that there is a subsequence of $\hat{v}_{\alpha}=\eta_{\delta}\left(\tau_{\alpha} x\right) v_{\alpha}$ where $\delta$ is as in the above lemma, that weakly converges in $\mathcal{D}^{1,2}\left(I R^{n}\right)$ to some function $v$, a weak solution on $\mathcal{D}^{1,2}\left(I R^{n}\right)$ to (3.14). Note that this time the singular term disappears because $x_{0} \neq p$ and because of course $t_{\alpha} \rightarrow 0$.

It remains to show that $v \neq 0$. For this purpose, take a point $a \in I R^{n}$ and a constant $r>0$ such that $|a|+r<r_{o} \tau_{\alpha}^{-1}$, where $r_{o} \in\left(0, \frac{\delta_{g}}{2}\right)$ is a constant such that inequality (3.9) is satisfied. Then, we have

$$
\exp _{x_{\alpha}}\left(\tau_{\alpha} B(a, r)\right) \subset B\left(\exp _{x \alpha}\left(\tau_{\alpha} a\right), C_{o} r \tau_{\alpha}\right),
$$

and

$$
\exp _{x_{\alpha}}\left(\tau_{\alpha} B\left(C_{o} r\right)\right)=B\left(x_{\alpha}, C_{o} r \tau_{\alpha}\right)
$$

$C_{o}$, here, is the constant appearing in inequality (3.9). Since we have

$$
\int_{B(a, r)}\left|\nabla v_{\alpha}\right|^{2} d v_{\tilde{g}_{\alpha}}=\int_{\exp _{x_{\alpha}}\left(\tau_{\alpha} B(a, r)\right)}\left|\nabla v_{\alpha}\right|^{2} d v_{g},
$$

we get by construction of $x_{\alpha}$ that for such $a$ and $r$,

$$
\int_{B(a, r)}\left|\nabla v_{\alpha}\right|^{2} d v_{\tilde{g}} \leq \lambda
$$

Suppose now that $v \equiv 0$. Take any function $h \in \mathcal{D}^{1,2}\left(I R^{n}\right)$ with support included in a ball $B(a, r) \subset I R^{n}$, with $a$ and $r$ as above. Then, by taking $\lambda$ small enough, we get by the same 
calculation done in (3.15) that $\int_{B(a, r)} \nabla \hat{v}_{\alpha} d v_{\tilde{g}}$ converges to 0 for all $a \in I R^{n}$ and $r>0$ such that $|a|+r<r_{o} \tau_{\alpha}^{-1}$. In particular,

$$
\int_{B\left(x_{\alpha}, t_{\alpha}\right)}\left|\nabla v_{\alpha}\right|^{2} d v_{g}=\int_{B\left(C_{0} r\right)}\left|\nabla v_{\alpha}\right|^{2} d v_{\tilde{g}} \rightarrow 0,
$$

which makes a contradiction. Thus $v \neq 0$.

The proof of the remaining statements of the lemma goes in the same way as in lemma 3.4 .

Proof of Theorem 3.1. First, it is worthy to mention that the value $G_{\infty}(v)$ taken on a nontrivial weak solution $v$ of the Euclidean equation (3.9) is greater or equal to the constant $\beta^{*}$. In fact, if $v$ is solution of (3.9), then by Hardy and Sobolev inequalities we have

$$
\int_{I^{n}}\left(|\nabla v|^{2}-h_{\infty}(p) \frac{v^{2}}{|x|^{2}}\right) d x=f(p) \int_{I^{n}}|v|^{2^{*}} d x \leq f(p) K^{2^{*}}(n, 2)\left(\int_{I R^{n}}|\nabla v|^{2} d x\right)^{\frac{2^{*}}{2}},
$$

and

$$
\int_{I R^{n}}\left(|\nabla v|^{2}-h_{\infty}(p) \frac{v^{2}}{|x|^{2}}\right) d x \geq\left(1-h_{\infty}(p) K^{2}(n,-2,2)\right) \int_{I R^{n}}|\nabla v|^{2} d x,
$$

then by (3.17) and (3.18) we get

$$
\begin{aligned}
G_{\infty}(v) & =\frac{1}{n} \int_{I R^{n}}\left(|\nabla v|^{2}-h_{\infty}(p) \frac{v^{2}}{|x|^{2}}\right) d x \\
& \geq \frac{\left(1-h_{\infty}(p) K^{2}(n,-2,2)\right)^{\frac{n}{2}}}{n f(p)^{\frac{n-2}{2}} K^{n}(n, 2)}=\beta^{*} .
\end{aligned}
$$

Now, let $u_{\alpha}$ be a sequence of solutions of $\left(E_{\alpha}\right)$ such that $\int_{M} f\left|u_{\alpha}\right|^{2^{*}} d v_{g} \leq C, u_{\alpha}$ is then a bounded Palais-Smale sequence of $J_{\alpha}$ at some level $\beta$. Up to a subsequence, we may assume that $u_{\alpha}$ converges weakly in $H_{1}^{2}(M)$ and almost everywhere in $M$ to a solution $u$ of $\left(E_{\infty}\right)$. Set $v_{\alpha}=u_{\alpha}-u$, then by Lemma 3.1, $v_{\alpha}$ is a Palais sequence of $J_{\alpha}$ at level $\beta_{1}=$ $\beta-J_{\infty}(u)+o(1)$. If $v_{\alpha} \rightarrow 0$ strongly in $H_{1}^{2}(M)$, then the theorem is proved with $k=l=0$. If $v_{\alpha} \rightarrow 0$ only weakly in $H_{1}^{2}(M)$, then we apply Lemmas 3.3, 3.4 and 3.5 to get a new PalaisSmale sequence $v_{\alpha}^{1}$ at level $\beta_{2} \leq \beta_{1}-\beta^{*}+o(1)$. So, either $\beta_{2}<\beta^{*}$ and then $v_{\alpha}^{1}$ converges strongly to 0 , or $\beta_{2} \geq \beta^{*}$ and in this case we repeat the procedure for $v_{\alpha}^{1}$ to obtain again a new Palais -Smale sequence at smaller level. By induction, after a number of iterations, we obtain a Plais-Smale sequence at a level smaller than $\beta^{*}$.

Corollary 3.1. Suppose that the sequence $u_{\alpha}$ of weak solutions of $\left(E_{\alpha}\right)$ is such that

$$
E\left(u_{\alpha}\right)=\int_{M} f\left|u_{\alpha}\right|^{2^{*}} d v_{g} \leq c \leq \frac{\left(1-h_{\infty}(p) K^{2}(n, 2,-2)\right)^{\frac{n}{2}}}{\left(\sup _{M} f\right)^{\frac{n-2}{2}} K^{n}(n, 2)} .
$$

Then, up to a subsequence, $u_{\alpha}$ converges strongly in $H_{1}^{2}(M)$ to a nontrivial weak solution $u$ of $\left(E_{\infty}\right)$. 
Proof. By Theorem 3.1, there is a weak solution $u$ of $\left(E_{\infty}\right)$ such that, up to a subsequence of $u_{\alpha}$, we have

$$
\begin{aligned}
u_{\alpha}= & u+\sum_{i=1}^{k}\left(R_{\alpha}^{i}\right)^{\frac{2-n}{n}} \eta_{\delta}\left(\exp _{p}^{-1}(x)\right) v_{i}\left(\left(R_{\alpha}^{i}\right)^{-1} \exp _{p}^{-1}(x)\right) \\
& +\sum_{j=1}^{l} f\left(x_{o}^{j}\right)^{\frac{2-n}{4}}\left(r_{\alpha}^{j}\right)^{\frac{2-n}{n}} \eta_{\delta}\left(\exp _{x_{\alpha}^{j}}^{-1}(x)\right) v_{j}\left(\left(r_{\alpha}^{j}\right)^{-1} \exp _{x_{\alpha}^{j}}^{-1}(x)\right)+\mathcal{W}_{\alpha}, \\
& \text { with } \mathcal{W}_{\alpha} \rightarrow 0 \text { in } H_{2}^{1}(M),
\end{aligned}
$$

and

$$
c \geq E\left(u_{\alpha}\right)=n J_{\alpha}\left(u_{\alpha}\right)=n J_{\infty}(u)+n \sum_{i=1}^{k} G_{\infty}\left(v_{i}\right)+n \sum_{j=1}^{l} f\left(x_{o}^{j}\right)^{\frac{2-n}{2}} G\left(v_{j}\right)+o(1) .
$$

Suppose that $u \equiv 0$, if there exists $i, 1 \leq i \leq k$ such that $v_{i} \neq 0$, then by (3.19) we get

$$
c \geq \frac{\left(1-h_{\infty}(p) K^{2}(n, 2,-2)\right)^{\frac{n}{2}}}{\left(\sup _{M} f\right)^{\frac{n-2}{2}} K^{n}(n, 2)},
$$

thus, $v_{i} \equiv 0, \forall i, 1 \leq i \leq k$, case in which Lemma 3.4 applies, that is, there exists $v_{j} \neq 0$ such that

$$
c \geq \frac{f\left(x_{o}^{j}\right)^{\frac{2-n}{2}}}{K^{n}(n, 2)}>\frac{\left(1-h_{\infty}(p) K^{2}(n, 2,-2)\right)^{\frac{n}{2}}}{\left(\sup _{M} f\right)^{\frac{n-2}{2}} K^{n}(n, 2)} .
$$

Hence, $u \neq 0$. Furthermore, $J_{\infty}(u)>0$, from which we can conclude that $k=l=0$. In particular, $u_{\alpha}$ converges strongly in $H_{1}^{2}(M)$ to $u$.

\section{References}

[1] D. Cao and S. Peng, A global compactness result for singular elliptic problems involving critcal Sobolev exponent, Transcation of AMS, 131(6) (2003), 1857-1966.

[2] O. Druet, E. Hebbey and F. Robert, Blow-Up Theory for Elliptic PDEs in Riemannian Geometry, Princeton University Press, 2004.

[3] M. Dellinger, Etude asymptotique et multiplicité pour l'équation de Sobolev Poincaré, Thesis, University of Paris VI, 2007.

[4] E. Hebey, Introduction à l'analyse non linéaire sur les variétés, Diderot, 1997.

[5] F. Madani, Le problème de Yamabe avec singularités et la conjecture de Hebey-Vaugon, Thesis, Université Pierre et Marie Curie, 2009.

[6] D. Smet, Nonlinear Schrödinger equations with Hardy potential and critical nonlinearities, Transactions of AMS, 357(7) (2004), 2909-2938.

[7] M. Struwe, A global compactnes result for elliptic boudary value problems involving limiting nonlinearities, Math. Z., 187 (1987), 511-517.

[8] S. Terracini, On positive entire solutions to a class of equations with a singular coefficient and critical exponent, Adv. Differential Equations, 1(2) (1996), 241-264. 